\title{
ON THE SUPPORTING-PLANE PROPERTY OF A CONVEX BODY ${ }^{1}$
}

\author{
DAVID MOSKOVITZ AND L. L. DINES
}

In an earlier paper, ${ }^{2}$ the authors have shown that in a linear space $\subseteq$ with an inner product, a set $\mathfrak{M}$ which is closed and linearly connected is supported at a set of boundary points which is everywhere dense on the boundary of $\mathfrak{M}$, and an example is given to show that such a set $\mathfrak{M}$ may have boundary points through which no supporting plane exists. The purpose of this paper is to show that if a set, in addition to being linearly connected and closed, also possesses inner points, then it is completely supported at its boundary points. In (I), reference was made to a paper by Ascoli in which such a result was obtained in a separable space. We do not assume our space $\subseteq$ to be separable. The definitions and results of (I) will be used in this paper.

A set $\Re$, which is a proper subset of the space $\mathfrak{S}$, will be called a convex body if it is linearly connected, closed, and possesses inner points. In the sequel $\Omega$ will always denote a convex body.

With reference to the set $\Re$, there is associated with each point $x$ of the space $\subseteq$ a nonnegative number $r(x):$ if $x$ is an inner point of $\Omega, r(x)$ is defined as the least upper bound of the radii of spheres about $x$ which do not contain points exterior to $\Omega$; for other points of $\mathfrak{S}, r(x)$ is defined to be zero. We will call $r(x)$ the radius at the point $x$.

If $x_{1}$ is a point of $\Omega$, all points $x$ of the sphere $\left\|x-x_{1}\right\| \leqq r\left(x_{1}\right)$ are points of $\Omega$.

TheOREM 1. Let $r_{1}$ and $r_{2}$ be the radii at the points $x_{1}$ and $x_{2}$, respectively, of the convex body $\Omega$. Then the radius $r$ at the point

satisfies

$$
x=x_{1}+k\left(x_{2}-x_{1}\right), \quad 0 \leqq k \leqq 1,
$$

$$
r \geqq r_{1}+k\left(r_{2}-r_{1}\right) .
$$

Proof. Let $y=x+\rho u$, where $\rho=r_{1}+k\left(r_{2}-r_{1}\right)$ and $\|u\|=1$. The points $y_{1}=x_{1}+r_{1} u$ and $y_{2}=x_{2}+r_{2} u$ are points of $\Omega$. But from the definitions of $x, \rho$, and $y$, it follows that $y=y_{1}+k\left(y_{2}-y_{1}\right)$. Hence $y$, being on the segment joining $y_{1}$ and $y_{2}$, is also a point of $\Omega$. Consequently

1 Presented to the Society, September 5, 1939.

2 On convexity in a linear space with an inner product, Duke Mathematical Journal, vol. 5 (1939), pp. 520-534. Hereafter, this paper will be referred to by (I). 
all points on the boundary of the sphere with radius $\rho$ and center $x$ are in $\Omega$. Since $\Omega$ is linearly connected, also all points within this sphere are in $\Re$. Therefore $r \geqq \rho$ and the theorem is established.

The following corollaries, which appear self-evident in ordinary space, can be shown to be direct consequences of the preceding theorem.

CoROllary 1. Each point of the segment joining two inner points of $\Omega$ is an inner point of $\Omega$.

CoROllaRy 2. If $x_{0}$ is a boundary point and $x_{1}$ an inner point of $\Omega$, then the points $x=x_{1}+k\left(x_{0}-x_{1}\right)$ are inner points of $\Omega$ for $0 \leqq k<1$, and exterior points for $k>1$.

With reference to a given boundary point $x_{0}$ of the set $\Re$, there is associated with each point $x$, other than $x_{0}$, of the space $\mathfrak{S}$ a nonnegative number $s(x)$, defined by ${ }^{3}$

$$
s(x)=r(x) /\left\|x-x_{0}\right\| .
$$

If $x$ is an exterior point or a boundary point of $\Omega$, other than $x_{0}$, $s(x)$ is equal to zero; if $x$ is an inner point of $\Omega, s(x)$ is positive; $s\left(x_{0}\right)$ is not defined.

It is also obvious that $s(x) \leqq 1$, since $r(x) \leqq\left\|x-x_{0}\right\|$.

THEOREM 2. Let $x_{0}$ be a given boundary point of the convex body $\Omega$, and let $x_{t}$ be given by

$$
x_{t}=x_{0}+t u, \quad \text { where } t>0,\|u\|=1 \text {. }
$$

Then, for fixed $u$,

(a) $s\left(x_{t}\right)$ is a non-decreasing function as $t \rightarrow 0$; and

(b) $\lim _{t \rightarrow 0} s\left(x_{t}\right)$ exists.

Proof. In case there are no points of $\Omega$ given by (1), the theorem is obviously true, for then

$$
s\left(x_{t}\right)=0 \text { for } t>0, \quad \lim _{t \rightarrow 0} s\left(x_{t}\right)=0 .
$$

In case there are points of $\Omega$ given by (1), let $x_{1}$ and $x_{2}$ be two points of $\Omega$ on (1) for parameter values $t_{1}$ and $t_{2}$, where $t_{1}<t_{2}$; then we have

$$
\begin{aligned}
x_{1} & =x_{0}+t_{1} u, & x_{2} & =x_{0}+t_{2} u ; \\
s\left(x_{1}\right) & =r\left(x_{1}\right) / t_{1}, & s\left(x_{2}\right) & =r\left(x_{2}\right) / t_{2} .
\end{aligned}
$$

${ }^{3}$ Since $s$ is a function of $x_{0}$ as well as $x$, a more explicit notation would be $s\left(x_{0}, x\right)$; but the simpler notation will suffice, inasmuch as the function is to be used in the sequel only with reference to a fixed boundary point $x_{0}$. 
But $x_{1}=x_{0}+\left(t_{1} / t_{2}\right)\left(x_{2}-x_{0}\right)$, and hence, by Theorem 1 , we have

$$
r\left(x_{1}\right) \geqq \frac{t_{1}}{t_{2}} r\left(x_{2}\right),
$$

since $r\left(x_{0}\right)=0$. Therefore, by (2), $s\left(x_{1}\right) \geqq s\left(x_{2}\right)$.

This result establishes part (a) of the theorem. Since $s\left(x_{t}\right)$ cannot exceed one, obviously part (b) of the theorem is true.

Let $\Sigma$ be the unit sphere about $x_{0}$; and let $p_{u}$ be the point on $\Sigma$ given by $p_{u}=x_{0}+u,\|u\|=1$. Let $x_{t}=x_{0}+t u,(0<t<1)$, be the segment joining $x_{0}$ to $p_{u}$; and let 4

$$
\sigma(u)=\lim _{t \rightarrow 0} s\left(x_{t}\right) .
$$

We thus have a function $\sigma(u)$ uniquely defined at each point $p_{u}$ on the sphere $\Sigma$. Obviously, by its definition, we have

$$
0 \leqq \sigma(u) \leqq 1 \text {. }
$$

Also $\sigma(u)=0$ only if the segment joining $x_{0}$ to $p_{u}$ does not contain any inner points of $\Omega$. If the segment joining $x_{0}$ to $p_{u}$ contains inner points of $\Re$, we have $\sigma(u)>0$.

LEMMA 1. Let $p_{u}$ and $p_{v}$ be two points on $\Sigma$, such that

$$
p_{u}=x_{0}+u, \quad p_{v}=x_{0}+v, \quad v=-u .
$$

Then at least one of the numbers $\sigma(u)$ or $\sigma(v)$ is equal to zero.

Proof. Assume $\sigma(u)>0$; then the segment joining $x_{0}$ to $p_{u}$ contains inner points. Consequently, by Corollary 2 , the segment joining $x_{0}$ to $p_{v}$ does not contain any inner points. Therefore, $\sigma(v)=0$.

THEOREM 3. Let $x_{0}$ be a given boundary point of the convex body $\Omega$, and let $\Sigma$ be the unit sphere about $x_{0}$. Let $p_{u}$ and $p_{v}$ given by

$$
p_{u}=x_{0}+u, \quad\|u\|=1, \quad p_{v}=x_{0}+v, \quad\|v\|=1
$$

be two distinct points on $\Sigma$, for which $\sigma(u)$ and $\sigma(v)$ are both positive. Then there exists a point $p_{w}$ distinct from $p_{u}$ and $p_{v}$ for which

$$
\sigma(w)>\frac{1}{2}[\sigma(u)+\sigma(v)] .
$$

4 The limit was shown to exist in Theorem 2; we are denoting the value of this limit by $\sigma(u)$. It may be of interest to note that

$$
\sigma(u)=\lim _{x \rightarrow x_{0}, \text { along } x_{t}=x_{0}+t u} s(x)=\lim _{x \rightarrow x_{0}} \frac{r(x)-r\left(x_{0}\right)}{\left\|x-x_{0}\right\|}=r_{u}^{\prime}\left(x_{0}\right)
$$

is the directional derivative of $r(x)$ at $x_{0}$ in the direction $u$. 
Proof. Let $x_{t}$ and $y_{t}$ be points of $\Omega$ given by

$$
x_{t}=x_{0}+t u, \quad y_{t}=x_{0}+t v, \quad 0<t<1,
$$

and let $((u, v))=\lambda$. Then, certainly $|\lambda| \leqq 1$. But if $\lambda=1, u=v$, and $p_{u}$ and $p_{v}$ are not distinct. If $\lambda=-1, u=-v$, in which case not both of the numbers $\sigma(u)$ and $\sigma(v)$ can be positive, because of Lemma 1. Consequently, we have

$$
-1<\lambda<1
$$

Let $z_{t}=\frac{1}{2}\left(x_{t}+y_{t}\right)$; then $z_{t}=x_{0}+\xi t w$, where $\|w\|=1$ and $\xi=\frac{1}{2}(1+\lambda)^{1 / 2}$. Thus

$$
0<\xi<1 \text {. }
$$

We thus have a point $p_{w}$ on the sphere $\Sigma$ defined by $p_{w}=x_{0}+w$. Now, $r\left(z_{t}\right) \geqq \frac{1}{2}\left[r\left(x_{t}\right)+r\left(y_{t}\right)\right]$, by Theorem 1 . Hence

$$
s\left(z_{t}\right)=\frac{r\left(z_{t}\right)}{\xi t} \geqq \frac{1}{2 \xi}\left[\frac{r\left(x_{t}\right)}{t}+\frac{r\left(y_{t}\right)}{t}\right]=\frac{1}{2 \xi}\left[s\left(x_{t}\right)+s\left(y_{t}\right)\right],
$$

and

$$
\lim _{t \rightarrow 0} s\left(z_{t}\right) \geqq \frac{1}{2 \xi} \lim _{t \rightarrow 0}\left[s\left(x_{t}\right)+s\left(y_{t}\right)\right]
$$

from which

$$
\sigma(w) \geqq \frac{1}{2 \xi}[\sigma(u)+\sigma(v)]>\frac{1}{2}[\sigma(u)+\sigma(v)] .
$$

Thus the theorem is established.

Let $\bar{\sigma}$ denote the least upper bound of the function $\sigma(u)$ as $p_{u}$ varies over the sphere $\Sigma$. Then, also $0 \leqq \bar{\sigma} \leqq 1$; and $\bar{\sigma}=0$ is possible only for sets which do not have any inner points. For a convex body $\Omega$, we have $0<\bar{\sigma} \leqq 1$.

In the material which follows, it is to be understood that $x_{0}$ is a fixed boundary point of the convex body $\Omega, s(x)$ is defined relative to $x_{0}, \Sigma$ is the unit sphere about $x_{0}, \sigma(u)$ is the function defined above on the boundary of $\Sigma$, and $\bar{\sigma}$ the least upper bound of $\sigma(u)$ on $\Sigma$.

TheOREM 4. If there is a point $p_{u}$ on $\Sigma$ for which $\sigma(u)=\bar{\sigma}$, this point is unique.

Proof. Suppose, if possible, that there were a second point $p_{v}$ for which $\sigma(v)=\bar{\sigma}$. Then, by Theorem 3 , since $\bar{\sigma}>0$, there would be a point $p_{w}$ for which 


$$
\sigma(w)>\frac{1}{2}[\sigma(u)+\sigma(v)]=\bar{\sigma} .
$$

But since no $\sigma(w)$ can exceed $\bar{\sigma}$, there cannot be a second point $p_{v}$ of the type described.

TheOREM 5. Let $p_{u}$ be a point on $\Sigma$ for which $\sigma(u)=\bar{\sigma}$. If $v$ satisfies the conditions $\|v\|=1$ and $((u, v))<0$, then the points $z_{t}=x_{0}+t v, t>0$, are exterior points of $\Re$.

Proof. Let $p_{u}=x_{0}+u,\|u\|=1$, and $p_{v}=x_{0}+v,\|v\|=1$; and let $((u, v))=-\lambda$, where $\lambda>0$. Assume, if possible, that there is a point $z=x_{0}+d v, d>0$, belonging to $\Omega$. Let $w$ be the projection (defined in (I)) of $z$ on the line through $x_{0}$ and $p_{u}$. Then

$$
w=p_{u}+c\left(x_{0}-p_{u}\right),
$$

where

$$
\begin{aligned}
c & =\frac{\left(\left(z-p_{u}, x_{0}-p_{u}\right)\right)}{\left\|p_{u}-x_{0}\right\|^{2}}=\left(\left(z-x_{0}-u,-u\right)\right) \\
& =((d v-u,-u))=1+\lambda d .
\end{aligned}
$$

Hence,

$$
w=p_{u}-(1+\lambda d) u=x_{0}-\lambda d u .
$$

On the segment joining $x_{0}$ to $p_{u}$, let $x_{t}=x_{0}+t u$ be an inner point of $\Omega$. Let $y_{t}$ be the projection of $x_{0}$ on the line through $x_{t}$ and $z$. Then

$$
y_{t}=x_{t}+k\left(z-x_{t}\right)
$$

where

$$
k=\frac{\left(\left(x_{0}-x_{t}, z-x_{t}\right)\right)}{\left\|z-x_{t}\right\|^{2}}=\frac{((-t u, d v-t u))}{\left\|z-x_{t}\right\|^{2}}=\frac{\lambda t d+t^{2}}{d^{2}+2 \lambda t d+t^{2}},
$$

since $z-x_{t}=z-x_{0}+x_{0}-x_{t}=d v-t u$ and

$$
\left\|z-x_{t}\right\|^{2}=d^{2}-2 t d((u, v))+t^{2}=d^{2}+2 \lambda t d+t^{2} .
$$

From the above value of $k$, it is easily seen that $0<k<1$, which means that $y_{t}$ is a point of $\Omega$. The following are easily established:

$$
\left\|y_{t}-x_{0}\right\|^{2}=\frac{t^{2} d^{2}\left(1-\lambda^{2}\right)}{d^{2}+2 \lambda t d+t^{2}} \neq 0
$$

since $\lambda \neq \pm 1$, and

$$
\|z-w\|^{2}=d^{2}\left(1-\lambda^{2}\right) .
$$


From these, and previous results, we obtain

$$
\frac{\left\|x_{t}-x_{0}\right\|^{2}}{\left\|y_{t}-x_{0}\right\|^{2}}=\frac{t^{2}\left(d^{2}+2 \lambda t d+t^{2}\right)}{t^{2} d^{2}\left(1-\lambda^{2}\right)}=\frac{d^{2}+2 \lambda t d+t^{2}}{d^{2}\left(1-\lambda^{2}\right)}=\frac{\left\|z-x_{t}\right\|^{2}}{\|z-w\|^{2}} .
$$

Therefore, we have

$$
\frac{\left\|x_{t}-x_{0}\right\|}{\left\|y_{t}-x_{0}\right\|}=\frac{\left\|z-x_{t}\right\|}{\|z-w\|} .
$$

Now $s\left(y_{t}\right)=r\left(y_{t}\right) /\left\|y_{t}-x_{0}\right\|$ and $s\left(x_{t}\right)=r\left(x_{t}\right) /\left\|x_{t}-x_{0}\right\|$, where $r\left(y_{t}\right)$ and $r\left(x_{t}\right)$ denote the radii at the points $y_{t}$ and $x_{t}$, respectively. Also $r\left(y_{t}\right) \geqq(1-k) r\left(x_{t}\right)$, by Theorem 1 and the definition of $y_{t}$. Hence

$$
\begin{aligned}
\frac{s\left(y_{t}\right)}{s\left(x_{t}\right)}=\frac{r\left(y_{t}\right)}{\left\|y_{t}-x_{0}\right\|} \cdot \frac{\left\|x_{t}-x_{0}\right\|}{r\left(x_{t}\right)} & \geqq(1-k) \frac{\left\|x_{t}-x_{0}\right\|}{\left\|y_{t}-x_{0}\right\|} \\
& =(1-k) \frac{\left\|z-x_{t}\right\|}{\|z-w\|},
\end{aligned}
$$

the last equality being a consequence of (3).

But $k=\left\|y_{t}-x_{t}\right\| /\left\|z-x_{t}\right\|$ and $1-k=\left\|z-y_{t}\right\| /\left\|z-x_{t}\right\|$. Therefore, from (4), we have

$$
s\left(y_{t}\right) \geqq \frac{\left\|z-y_{t}\right\|}{\|z-w\|} s\left(x_{t}\right) .
$$

Now,

$$
\lim _{t \rightarrow 0} \frac{\left\|z-y_{t}\right\|}{\|z-w\|}=\frac{d}{d\left(1-\lambda^{2}\right)^{1 / 2}}=\frac{1}{\left(1-\lambda^{2}\right)^{1 / 2}}>1,
$$

since $z$ and $w$ are independent of $t$, while $y_{t} \rightarrow x_{0}$ as $t \rightarrow 0$. Therefore, from (5),

$$
\lim _{t \rightarrow 0} s\left(y_{t}\right) \geqq \frac{1}{\left(1-\lambda^{2}\right)^{1 / 2}} \sigma(u)>\sigma(u)=\bar{\sigma} .
$$

But this is impossible; hence the assumption that $z$ was a point of $\Omega$ is untenable.

Theorem 6. Let $p_{u}$ be a point on $\Sigma$ for which $\sigma(u)=\bar{\sigma}$. Then the plane

$$
\pi(x) \equiv\left(\left(u, x-x_{0}\right)\right)=0
$$

is a supporting plane of $\AA$ through the boundary point $x_{0}$.

Proof. If the plane (6) were not a supporting plane, there would be 
a point $z$ of $\Re$ for which $\pi(z)<0$. Let $v=\left(z-x_{0}\right) /\left\|z-x_{0}\right\|$; then

$$
((u, v))=\frac{\pi(z)}{\left\|z-x_{0}\right\|}<0, \quad z=x_{0}+\left\|z-x_{0}\right\| v .
$$

But, $v$ satisfies the conditions of Theorem 5 ; therefore $z$ must be an exterior point of $\Omega$. Consequently, there cannot be a point $z$ of $\Omega$ for which $\pi(z)<0$; and (6) is indeed a supporting plane.

THEOREM 7. Let $x_{0}$ be a given boundary point of the convex body $\Omega$, and let $\Sigma$ be the unit sphere about. $x_{0}$. There is a unique point $p_{u}^{-}$on $\Sigma$ for which $\sigma(\bar{u})=\bar{\sigma}$.

Proof. We have only to show the existence of one point $p_{u}^{-}$for which $\sigma(\bar{u})=\bar{\sigma}$. The uniqueness of this point will follow from Theorem 4.

From the definition of $\bar{\sigma}$ it follows that for any preassigned $\epsilon>0$, there exists a point on $\Sigma$ for which the value of $\sigma$ is greater than $\bar{\sigma}-\epsilon$. Choose a monotone decreasing sequence of positive numbers $\left\{\epsilon_{n}\right\}$ with limit zero. Corresponding to each $\epsilon_{n}$ there exists a point $p_{u_{n}}$ on $\Sigma$ for which $\sigma\left(u_{n}\right)>\bar{\sigma}-\epsilon_{n}$. We wish to show that the sequence of points $\left\{p_{u_{n}}\right\}$ on $\Sigma$ converges.

Let $p_{u_{n}}=x_{0}+u_{n},\left\|u_{n}\right\|=1$, and $p_{u_{m}}=x_{0}+u_{m},\left\|u_{m}\right\|=1$. Then

$$
\left\|p_{u_{n}}-p_{u_{m}}\right\|^{2}=2-2\left(\left(u_{n}, u_{m}\right)\right) \text {. }
$$

Let $w=\frac{1}{2}(1 / \xi)\left(u_{n}+u_{m}\right)$, where $\xi$ is so chosen that $\|w\|=1$. Then we have

$$
\xi^{2}=\frac{1}{2}\left[1+\left(\left(u_{n}, u_{m}\right)\right)\right] .
$$

Let $p_{w}=x_{0}+w$; from the proof of Theorem 3, we know that

$$
\sigma(w) \geqq \frac{1}{2 \xi}\left[\sigma\left(u_{n}\right)+\sigma\left(u_{m}\right)\right]>\frac{1}{2 \xi}\left[2 \bar{\sigma}-\epsilon_{n}-\epsilon_{m}\right] .
$$

But $\bar{\sigma} \geqq \sigma(w)$; hence $\bar{\sigma}>(1 / \xi)\left[\bar{\sigma}-\left(\epsilon_{n}+\epsilon_{m}\right) / 2\right]$, from which

$$
\xi^{2}>\left[1-\frac{1}{2 \bar{\sigma}}\left(\epsilon_{n}+\epsilon_{m}\right)\right]^{2}
$$

Using the value of $\xi^{2}$ from (8) we easily find that

$$
\left(\left(u_{n}, u_{m}\right)\right)>2\left[1-\frac{1}{2 \bar{\sigma}}\left(\epsilon_{n}+\epsilon_{m}\right)\right]^{2}-1 .
$$

Then using (7), we obtain 


$$
\left\|p_{u_{n}}-p_{u_{m}}\right\|^{2}<\frac{4}{\bar{\sigma}}\left(\epsilon_{n}+\epsilon_{m}\right)-\frac{1}{\bar{\sigma}^{2}}\left(\epsilon_{n}+\epsilon_{m}\right)^{2} .
$$

Since $\lim _{n, m \rightarrow \infty}\left\|p_{u_{n}}-p_{u_{m}}\right\|=0$ and the space $\subseteq$ is complete, as was assumed in (I) and throughout this paper, the sequence $\left\{p_{u_{n}}\right\}$ converges to a point $p_{\bar{u}}$. This point $p_{\bar{u}}$ is on $\Sigma$, and moreover $\sigma(\bar{u})=\bar{\sigma}$, since it is easily shown that $\sigma(\bar{u})$ is greater than $\bar{\sigma}-\epsilon$ for any preassigned positive $\epsilon$.

Theorem 8. A convex body $\Omega$ is completely supported at its boundary points.

Proof. Let $x_{0}$ be a boundary point of $\Omega$. There exists a point $p_{u}$ on the unit sphere $\Sigma$ about $x_{0}$ for which $\sigma(u)=\bar{\sigma}$, by Theorem 7 . Hence the plane $\left(\left(u, x-x_{0}\right)\right)=0$ is a supporting plane of $\AA$ through $x_{0}$, by Theorem 6 . Since similar statements can be made for each boundary point, $\Omega$ is completely supported at its boundary points.

From the material above, the following additional result may be established without much difficulty:

COROLlaRy 3. There exists a unique supporting plane through the boundary point $x_{0}$ of the convex body $\Omega$ only if $\bar{\sigma}=1$; for $\bar{\sigma}<1$, there is an infinite number of supporting planes through $x_{0}$.

A primary classification of boundary points of a convex body may thus be made in terms of $\bar{\sigma}$, which is a function defined over the boundary of the convex body.

Carnegie Institute of Technology 